\title{
Bifidobacterium animalis/Lactobacillus rhamnosus Probiotic Supplement
}

\author{
National Cancer Institute
}

\section{Source}

National Cancer Institute. Bifidobacterium animalis/Lactobacillus rhamnosus Probiotic

Supplement. NCI Thesaurus. Code C129649.

An orally bioavailable, probiotic supplement containing the non-pathogenic microorganisms Bifidobacterium animalis and Lactobacillus rhamnosus subspecies lactis with potential immunomodulating, anti-diarrheal and mucosal protective activities. Upon oral ingestion, the naturally-occurring bacterial components in Bifidobacterium animalis/Lactobacillus rhamnosus subspecies lactis probiotic supplement may improve digestion and help to maintain adequate colonization of the gastrointestinal (GI) tract by modulating the composition of the normal microflora. They produce lactic acid, hydrogen peroxide and other substances during fermentation, thereby creating an acidic environment that is unfavorable for pathogens. During colonization in the Gl tract, the bacteria may also form a protective intestinal barrier, which may prevent damage to the mucosal epithelium, maintains the integ rity of the epithelial barrier from potential damage by toxins and certain chemicals, and reduces the potential for attachment of pathogens. This further protects against bacterial translocation and infection. In addition, this agent may reduce the secretion of pro-inflammatory cytokines and may potentiate natural and acquired immunity. Altogether, this may increase immunity, prevent Gl mucosal damage, malabsorption, inflammation, and reduce diarrhea, such as diarrhea induced by inflammation and GI mucosa-damaging agents. 